\title{
Cloud-based loT Analytics for the Smart Grid: Experiences from a 3-year Pilot
}

\author{
T. Hasan, P. Kikiras, A. \\ Leonardi \\ AGT International \\ Hilpertstraße 35 \\ 64295 Darmstadt, Germany \\ \{thasan, pkikiras, ale- \\ onardi\}@agtinternational.com
}

\author{
H. Ziekow* \\ Furtwangen University \\ Robert-Gerwig-Platz 1 \\ 78120 Furtwangen, Germany \\ zie@hs-furtwangen.de
}

\author{
J. Daubert \\ TU Darmstadt / CASED \\ Mornewegstr. 32 \\ 64293 Darmstadt, Germany \\ joerg.daubert@cased.de
}

\begin{abstract}
The transformation of electrical grids into smart-grid is seen as one of the major technological challenges of our times and at the same time as one of the key domains for Internet of Things (IoT). Smart-home technologies and corresponding analytics are an integral part of many use cases in this field. In this paper we present a cloud-based test bed for capturing and analyzing smart-home data and report on experiences from a 3 year pilot with a cloud-based system. We discuss on real-world challenges that we encountered throughout the pilot - e.g. related to big data volumes and data quality and describe corresponding technical solutions.
\end{abstract}

\section{Categories and Subject Descriptors}

H.4 [Information Systems Applications]: Miscellaneous

\section{General Terms}

Internet of Things

\section{Keywords}

Smart-home, IoT, Analytics

\section{INTRODUCTION}

In recent years, environmental and economic considerations have fueled investments in renewable energy sources. For instance, member states of the European Union have set the so-called 20-20-20 goals, to obtain at least 20 percent of their electricity from renewable sources by 2020. Germany even strives for 80 percent renewable sources in the energy mix by 2050 . However, this change in the energy supply has severe implications on the operation of electrical grids and the corresponding ICT infrastructure. Decentralized

* Main part of the work was done while at AGT International. production (e.g. with solar panels on roof tops) causes the need to expand grid management infrastructures to the low voltage level, i.e. the level of streets and houses. However, it remains an open question how technical solutions for grid management of on this local level should be designed.

Smart-home technology is seen as a key enable for better understanding and managing the energy consumption on in the low voltage grid. To better understand the corresponding challenges and solutions for data capturing and processing, the German federal government has provided funding for the PeerEnergyCloud research project [12]. This paper reports on a cloud-based test bed for capturing and analyzing sensor data from smart-homes that was developed and piloted throughout this project. The pilot was conducted using smart-home hardware packages with sensors for capturing device specific energy consumption as well as sensors for environmental conditions (e.g. room temperature). In total we used 60 packages that were installed in various setups and different homes. Overall, we observed installations for up to three years and captured about 12 Billion sensor measurements in total. The emphasis of this paper is on the technical solutions that we developed for capturing and analyzing the data as well as on the challenges that we faced in the real-world deployment. Key contributions of the paper are the following:

- We provide a solution architecture and details on technical components for a test bed that supports cloudbased service on top of smart-home sensors.

- We discuss the real-world challenges for data capturing and analysis that we derived from a 3 year deployment in the smart home/smart grid domain.

- We present technical solutions that respond to the challenges that we encountered throughout the pilot.

The remainder of the paper is structured as follows. Section 2 discusses relevant related work. Section 3 provides a high level overview of the solution architecture and different components of our test bed and Section 4 discusses operational issues that we faced when piloting the system. The subsequent sections address technical details of the key components in our test bed and the mechanisms for addressing the 
encountered challenges. Section 5 drills down into the implementation of the gateway component that facilitates sensing within homes. Section 6 describes specific monitoring components that we developed for the smart-home domain. Section 7 presents analytics components that we developed for supporting smart-grid applications and discusses how we addressed the corresponding big data challenges. Section 8 presents end-user services on top of our test bed along with a privacy policy engine that we developed. Finally, we conclude the paper and discuss future work in Section 9.

\section{RELATED WORK}

Several systems integrating sensor networks with energy management systems at the consumer premises have been proposed so far.

The closest to ours project is Linear [9], a Flemish Smart Grid project focusing on solutions to match residential electricity consumption with available wind and solar energy, an approach referred to as demand response. Linear selected and deployed 2 types of smart appliances. The first type consists of postponable appliances, such as dishwashers, washing machines and tumble dryers, 445 of which were deployed in the Linear pilot initiative. The second type consists of buffered appliances, of which Linear included 15 domestic hot water buffers and 7 electrical vehicles. In the field test, 110 houses were equipped with smart meters. In each Linear household a home gateway communicated with the measurement devices and smart appliances and sent all collected data in real-time to the backend of the gateway provider. The data was then forwarded to the Linear pilot backend. The project suffered from the amount of data and transactions collected since no big data technologies to handle that were used. Additionally, in-house communications were one of the major sources of technical malfunctions. Linear, installed on average 11 ZigBee plugs in each home, half of them serving exclusively to bridge communication signals. Linear preferred "Ethernet over PLC to Wi-Fi" for connecting the different modules in the house with the gateway. This choice quite often caused conflicts with existing applications such as the home network and digital television. As a result, Linear technicians repeatedly needed to reconfigure and test the complete in-house communication setup. According to the Linear support team "Some families were so excited about the possibilities of the Home Energy Management System that they started moving plugs to different locations in order to trace standby losses, not being aware that they were messing up the network to the extent that the fridge plug started showing the behavior of a television in our databases". Similar issues have been experienced also in our pilot and in one of the following sections we will present our mitigation approach.

In [5] the authors evaluate the performance of an in-home energy management system based on a ZigBee wireless sensor network. The focus there is the performance of the system on the application level, meaning the investigation of the potential of both energy management and demand management. The evaluation that has been performed by the authors is based on simulation results, and not on real network deployments in households as in our work.

Similarly, in [14] energy management in homes has been

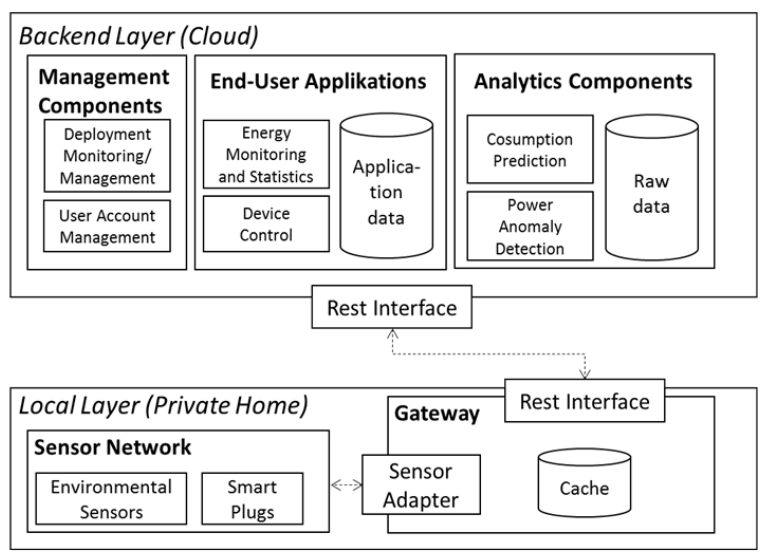

Figure 1: High-level architectural overview of the PEC ecosystem

investigated on a pilot consisting of only 3 households in Sacramento. The solution has been implemented using offthe-shelf components based on power line communication, and it included a web-based monitoring and control of home appliances.

\section{OVERVIEW OF SYSTEM ARCHITECTURE}

In this section we provide an overview of the main components of our testbed and the design decisions that drove the architecture. Key components are further detailed in sections 5, 6 and 7. We developed the testbed to support piloting of smart building technologies for delivering value-added services to end-users as well as for supporting grid operations through advanced analytics. Thus, the architectural design was driven by the need to (a) integrate sensors deployed in private homes, (b) deliver cloud-based services to end-users, and (c) support big data analytics over captured sensor data. Figure 1 gives an overview of the system architecture that supports these needs. In the architecture we distinguish two main physical layers: the local layer (hosted within private homes) and the backend layer (hosted in an cloud environment).

\subsection{Local Layer}

The main design goal of the local layer is to provide an easy to use solution for installing and connecting sensors in private homes to the backend infrastructure. Easy setup is a key requirement because persons with little technical experience should be able to make installations in their homes.

Our solution for the local layer comprises wireless sensors and a gateway component that we deploy in private homes. The sensors are smart building sensors that capture electricity consumption of individual devices and some context information within the home (e.g. room temperature and brightness). In addition, some sensors act as actuators that can control attached devices (i.e. switch power on or off). Several vendors offer sensors for smart buildings, using a range of different protocols (e.g. [1,2]).

By design our system supports a multitude of different sen- 
sor types and communication protocols. For the pilot we choose ZigBee based sensors as a solution that enables wireless deployment and coverage of larger houses through multihop communication.

When deployed in a house, the sensors establish a house specific network (i.e. with user specific network ID and encryption) and connect to the gateway component. The main task of the gateway is to bridge between the smart building sensors and the backend. It captures the arriving sensors data, buffers them, and forwards them via REST calls to a receiving component in the backend. In addition, the gateway provides a REST interfaces, allowing the backend to issue ad-hoc queries for sensor data, meta data, and to issue actuation commands.

The gateway software is based on Java and OSGi, making it compatible with a wide range of hardware platforms. Within the pilot we used media PCs to run the gateway but more lean hardware platforms are viable alternatives. ZigBee communication is enabled via a USB dongle and communication to the backend is established via the Internet. The network parameters for the ZigBee communication and for connecting to the backend are preconfigured when the components are shipped to the users. Through this preconfiguration, the installation on site is limited to deploying the sensors and connecting the gateway to an Internet router, thus is executable without detailed technical knowledge. All communication to the backed in encrypted via a VPN that the gateway connects to during startup. Besides encryption, this setup has the benefit of enabling secured communication from the backend to the gateway past firewalls and routers.

\subsection{Backend Layer}

The backend is hosted in a private cloud infrastructure. The main purposes of the backend are (1) collection of sensor data, (2) provisioning of web-based value added services to end-users, (3) provisioning of analytics for grid operations. Figure 1 shows the high level components that we roughly group into three sets. We refer to one set as Management Components that support the management of software and hardware deployments in the testbed. We refer to a second set of components as End-User Applications. These components provide end-user facing services and connectivity. A third set of components is called Analytics Components that provides storage and processing capabilities for big data analytics. The Management, End-User Applications, and Analytics Components, have different non-functional requirements, leading to different technology choices for their implementation.

The Management Components are used to manage and monitor the infrastructure. This includes health monitoring of all system components as well as management of user accounts and metadata about the deployments. In general, monitoring of the system health and management of user accounts is only moderately complex and can be implemented with standard approaches. However, the management of deployment metadata in an IoT context poses significant challenges and calls for special techniques. Metadata include information such as the association of a sensor to the object that it senses (e.g. an energy sensor attached to a fridge). In an uncontrolled environment - such as private homes - deployments might change without an explicit notification to the system (e.g. users redeploy smart plugs without updating the meta data). We address this challenge with dedicated analytics which implement a concept that we call "self-conscious sensor". Details about this concept and our solution are given in Section 6.

The End-User Applications provide users with statistics of their energy consumption and means to control (switch) their devices via web interfaces. Key requirements for these components are related to a good user experience in terms of system responsiveness. The challenge is to timely process the high resolution data from the sensors and to provide the results via an interactive interface. We address this challenge by leveraging concepts from the lambda architecture [10]. That is, we build the end-user applications on top of a database that acts as a serving-layer and feed the database via a dedicated batch-layer and speed-layer. The batch- and speed layer preprocess and aggregate the data for presentation. The serving layer only holds the subset of the complete sensors data which are needed for the end-user application as well as pre-computed aggregated statistics. This reduction of the dataset and pre-aggregation keeps the serving layer reasonably small and supports fast response times for end-user applications that query the data.

The requirements for the Analytics Components are mainly driven by (a) the large data volumes and (b) the support for exploratory data analysis. For every household in the pilot we receive about 1.2 million sensor measurement per day. This calls for storage and processing technologies that can handle big data. The batch layer provides a scalable storage that holds all raw sensor data and holds components for distributed batch processing (e.g. including a MapReduce framework). The speed layer leverages technologies of complex event processing that keep the recent incoming data in memory and run analytics over the live data streams. This design enables us to handle large data volumes in our analytics. It also addresses the need for flexible explorative data analytics by keeping the complete raw dataset and thus supporting the addition of arbitrary new analytics functions. More details about the implementation of the Analytics Components in Section 7.

\section{OPERATIONAL ISSUES IN THE PILOT}

Within the pilot we encountered several operational challenges while running our testbed. This section summarizes the key challenges and gives an overview of the learnings. The main challenges were related to two aspects: (1) connectivity issues, (2) and the uncontrolled environment for the sensor deployment. Connectivity issues occurred on two levels. One is the connection of ZigBee devices to the gateway and the other is the Internet connection from the gateway to the backend. The ZigBee connectivity heavily depended on the conditions within the various households. That is, the material of the wall and the distance between sensors (i.e. number of stories) had a strong impact on the connection quality. We managed to support deployment in large households by leveraging the multi-hop capabilities of the ZigBee protocol and by carefully selecting deployment positions (i.e. plugs in stairways between stories). Connectivity issues to the backend resulted from problems with some local inter- 


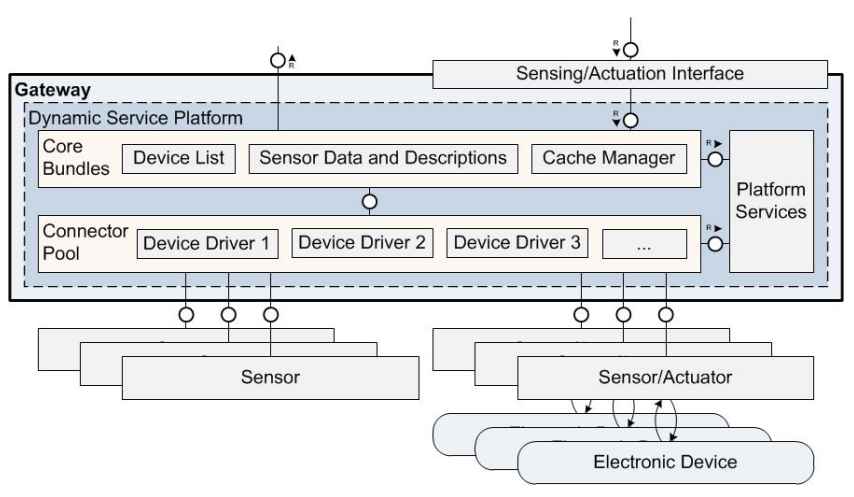

Figure 2: Gateway components.

net connections and downtimes of the backend. While loss of live data cannot be avoided, we implemented a solution to fill in resulting data gaps in the master data set of the backend. This is, we persisted recorded data on the local gateways and updated the backend as soon as the Internet connection was re-established (see Section 5 for details).

The challenge of sensing uncontrolled environment is inhered to the application domain of smart homes. The tested had to cope with a range of distortions caused by users. Examples of such distortions include accidental disconnection of the gateway (e.g. during cleaning) and rearranging of sensors. Such distortions are inherent and cannot be prevented by the system. Hence, the system must be able to adapt. We addressed this by extended monitoring capabilities and analytics which enabled the system to be aware of unintended changes in the deployment. Section 6 provides more details about the specific monitoring solutions.

\section{GATEWAY COMPONENTS}

The gateway provides an interface between the sensor network and the rest of the system. This means not only bridging the gap between the likely short-range network used by the sensors, but also the device-specific details and the higher-level, device-agnostic middleware in the backend [3].

The proposed gateway provides an abstraction layer which removes the device-specific details of the sensors and offers the gathered data in a standardized, device-independent way. The gateway also handles the management of the devices which are attached to it. For example, managing the registration of new devices, providing security and fault detection. The gateway's functionality should be extensible during the deployment lifetime of the gateway and the gathered data should also be stored in a cache on the gateway for optimization purposes. The gateway design is split into three main components: Core Bundles, Connector Pool and Platform Services.

\subsection{Core Bundles}

The Core Bundles perform the majority of tasks carried out by the Gateway. The set of Core Bundles comprises of the following components:
- The Device List, which handles joins and leaves of the devices to and from the sensor network so that the registration and subsequent de-registration of devices can be pushed to the backend.

- Sensor Data and Descriptions: the attached sensors need to be described in a device-independent way so that the backend can utilize the data and descriptions without the need of having explicit knowledge of the underlying implementation details of the various sensors.

- Cache Manager: to defend against failures of network connectivity, the gateway needs to cache sensor data temporarily until connectivity has been restored. The data needs to be kept in a cache for a pre-determined amount of time before being removed.

\subsection{Connector Pool}

The Connector Pool is where the device-specific protocol adaptors reside. The Connector Pool should have a number of device-specific protocol adaptors so that a number of different devices using different networking technologies can be attached to the gateway in a modular fashion. Each protocol adaptor should completely encapsulate the devicespecific details of the sensor, leaving the rest of the gateway to operate in as a generic way as possible. The protocol adaptor will handle the actual interface to the sensor network in order to receive data and all associated additional device-specific functionalities such as sending commands to the sensors for both actuation (where available) and management purposes.

\subsection{Platform Services}

Platform Services are generic house-keeping services to manage the Gateway as a service platform. In order for the gateway to be upgraded during its deployment lifecycle, it must be implemented in a modular way. These modules must be "hot pluggable", meaning that they can be dynamically upgraded without having to stop the running system. In order to achieve that, a set of Platform Services must be present providing an appropriate system and service platform which enables such hot plugging of software components.

\subsection{Gateway Implementation}

In order to meet the requirements described in the previous Section and satisfy the modularity and extensibility needs, we chose to build the gateway using the OSGi service framework. OSGi provides a platform where Java modules (called bundles in OSGi) can be dynamically installed, stopped, started, updated and uninstalled. By offering a serviceoriented architecture, bundles can register themselves as services, discover existing services and bind to listening services. These factors together mean that the functionality of the gateway can be modified during its deployment life-cycle thereby meeting our requirements. We created the following services in Java on top of OSGi:

The XBee protocol driver implements the XBee specific driver and performs the following functions:

- Device Discovery: it discovers XBee devices in the network. 
- Data handling: data is received from the attached devices and the payload is parsed and sent to the Cache Manager and also to the Data Push Interface so that the data can be forwarded to the backend for further processing.

- Actuation: if an application wishes to switch a device on or off then the XBee protocol driver creates the appropriate message to be sent to the device so that it can be activated or deactivated.

The Device List provides a dynamically updated list of attached devices which can be queried by the backend and thereby also provided to the applications. The Device List contains meta-data about each sensor that is attached, for example, its MAC address, type and if it is a power plug, whether or not its relay is switched on or off.

The Sensor Data and Descriptions are a set of high-level descriptions of the sensors themselves and the data they collect. The sensor descriptions are used by the gateway to keep track of which sensors are attached to the gateway at any particular time as we typically have sensors of multiple types in a deployment. The sensor data descriptions are used to convert the data sent by the sensors into a format which can be used by the backend and thereby the rest of the system.

When a message arrives from a sensor, it is processed by the gateway and the relevant data is extracted. A single sensor device can send messages with different types of data as it may have multiple sensors on-board (e.g., temperature, light, humidity, etc.) so the gateway needs to be aware of the different types of data that can be expected from the sensors.

\begin{tabular}{l} 
Sensor Data 1 Unformatted sensor data \\
\hline BRI $=3911 \mathrm{x}$ \\
TEM $=26.8 \mathrm{C}$ \\
BAT $=0 \mathrm{~K}$ \\
UBAT $=4.72 \mathrm{~V}$
\end{tabular}

The Sensor Data 1 shows an example of the unformatted sensor data as it arrives from the sensor at the gateway. The gateway parses this data and creates a internal representation of this data using the sensor data itself, information from the packet header and the time and date when the packet has been received.

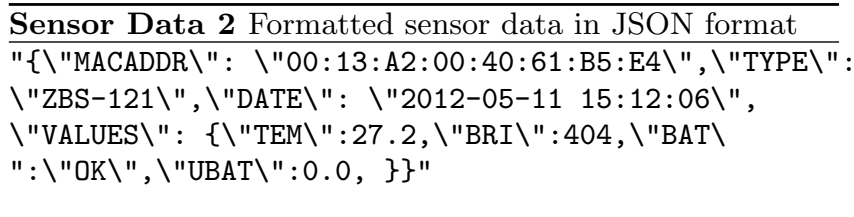

This internal representation is then converted into a JavaScript Object Notation (JSON) stanza which is then sent to the backend for processing. An example of the JSON stanza is shown in the Sensor Data 2.

The Cache Manager interacts with a local database (e.g., PostgreSQL) to temporarily store the gathered sensor data.
The Database is also accessible via the Sensing/Actuation Interface so that the applications can access the cache if necessary.

The Representational State Transfer (REST) Interface is the implementation of the interfaces used by the gateway to interact with the backend. It provides the following features: get the latest data from the database for a specific device, turn the relay of a device on or off (actuation capabilities) and send gathered data from a sensor to the Backend. The REST Interface is implemented using the JAX-RS framework and formats the payload of the REST calls in a JSON format.

\section{MONITORING AND SUPPORT SERVICE}

In this Section we describe two important components related to the management of the system, the monitoring and the plug change detection, respectively. These two components make the system itself "self-conscious" about its status and possible misconfiguration caused by the users.

\subsection{System state monitoring}

As we have to deal with a complex distributed system with many potential error sources, the state of the system has to be monitored constantly to be able to quickly detect faulty or abnormal behavior. For that, a number of services were implemented to help the administrators keep an overview. As the deployment consists of gateways connected through a VPN to a set of backend servers, it is obvious that it is crucial to monitor each part to a certain extent. Therefore, these three points for monitoring have been identified and covered in our deployment.

As implemented in our deployment, an alert can be raised when the number of connected sensors to a specific gateway drops, hinting at an unstable local ZigBee network or other problems with the gateway software.

As the VPN connection is an important part in the communication infrastructure, it has to be monitored as well. Services that dispatch alerts when new gateways connect or disconnect are needed. Therefore, in our case, gateways send an email whenever they connect to the VPN to be aware of newly set up installations or unexpected restarts of gateways. For manual inspection, there is a script that lists all connected gateways.

Furthermore, the responsiveness and availability of the backend servers themselves are crucial. To monitor the servers, we implemented services that look for recent activity in the databases. If the data is not written through to the database immediately, it can hint at problems with the REST-Interface or problems with handling the high amount of incoming REST calls and the resulting database writings. Because of pilot project character, it was of advantage to periodically restart the VMs to eliminate all stray or "buggy" processes that might have been initiated by faulty automatic calls of the REST interface or other components.

The majority of our services were implemented as scripts that run on the central backend servers and are either called 
manually or on a daily basis by e.g. a cron job. It came to our attention that sending automatically generated status emails through local SMTP server instances to a number of interested administrators is very useful for keeping a general overview of the system.

\subsection{Plug change detection}

Due to the loose installation of sensors and the fact that private homes are very dynamic environments, we could observe that users tend to switch sensors or connect new devices to their system without providing the information to the system. Any analytical component working with data from a household where the configuration has changed will yield wrong results. Therefore, we designed a component that can take required countermeasures to ensure the consistency of the data.

We implemented a scalable framework (based on Apache Storm and the machine learning framework Weka) that can prompt the user to confirm a detected configuration change or automatically trigger recalculation of any analytics models [8]. For this, it aggregates the consumption measurements for each device of a specific household over 24 hours and extracts a set of features from this aggregated data. With feature vectors from multiple days and from all devices of a specific household, two kinds of machine learning models are learned. The first is a classifier that accurately classifies feature vectors extracted from newly incoming data as one of the devices the model has been learned with. By having such a mechanism working with a high accuracy, (cyclic) swaps can be/are detected easily. However, for the case that we want to detect previously unknown devices, this approach gives only limited possibilities to asses the confidence with which newly collected data really comes from a device that is known to the household model, as a classifier will always chose one category, be it fitting or not. Therefore, we rely on a second model that clusters the data into as many clusters as there are sensors in the smart home kit and look at the distance of a newly incoming feature vector to the closest cluster center. If this distance is above a certain threshold, we can accurately assume that the data the feature vector has been extracted from is not coming from a usually attached device. For the first component we use statistical features like minimum (non-zero), maximum and average daily consumption among others. In addition to that, a histogram of the daily consumption data is computed and put into the feature vector as is. For the second component it suffices to only rely on the statistical features.

As stated, the performance of the swapping detection is inevitably linked with the performance of the classification mechanism. Our system is able to detect the devices with an accuracy of $97.08 \%$ and therefore also detect cyclic swaps very accurately.

When it comes to evaluation of the second model, we have to look at two parameters of the setup, namely the number of days of training data per device in the training set and the cluster distance threshold that determines the distance to the closest cluster center after which the device shall be detected as unknown.

In order to be able to evaluate this model appropriately, we
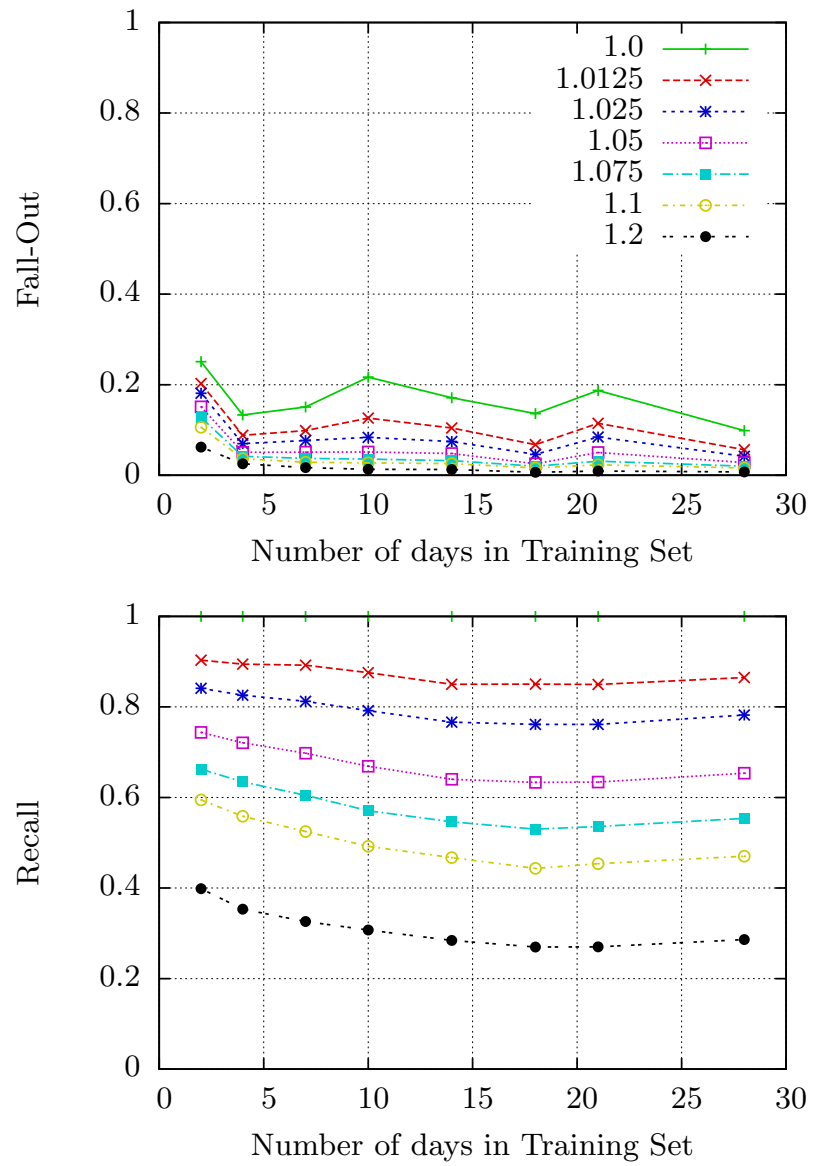

Figure 3: ClusterDist-based decision over number of days in training set and varying threshold $T_{\text {clusterDist }}$

furthermore need metrics tailored to the specific problem, providing the possibility of tuning the system towards the intended performance. Therefore, a short description of the chosen metrics shall be given at this point. We identified two questions that are of high interest in our scenario: How many of the unknown devices are we able to detect? And how many false alarms are issued by the system? The metric addressing the first question is the Recall. In this context, the Recall describes how many of the unknown instances in the test set really were recognized as such. The second measure is Fall-out. It describes how many of the tested known devices have been incorrectly classified as unknown devices, leading to a false alarm. It is key to maximize Recall and minimize Fall-out.

As can be seen in Figure 3, increasing the threshold $T_{\text {clusterDist }}$ we can observe characteristics one can expect, i.e. lowering recall and increasing fall-out. Furthermore it can be seen that with a rising number of instances per device in the training set, the Fall-out drops and many of the threshold combinations furthermore provide a constantly high Recall rate. It is now key to find a combination of number of days in training data and decision threshold that suits the application scenario. With a lower Recall, more unknown devices will falsely not be detected and with a too high Fall-out we get more false alarms. With a lower number of required days 
in the training set the system requires less time to set itself up to operate but will possibly work less robustly.

When taking a look at the application scenario of prompting the user for confirmation of changes made to the smart home environment, a high fall-out poses a problem. It can be perceived bothering or even disturbing to the user if he gets gets prompted on a too frequent basis. Therefore, we propose to mitigate this issue by setting a minimum number of consecutive days where an instance representing the behavior of an appliance at that day has to be detected as unknown under utilization of the same model. In the second scenario, where the detection of an unknown device triggers recalculation of an ML model, the false alarm rate is not as harmful, as an adequately designed system should be able to cope with a moderate amount of recalculations. In this case, the parameters can be chosen so that we have a high detection probability and an amount of false alarms that causes still manageable overhead.

\section{ANALYTICS COMPONENTS}

A central aspect of the pilot was to provide analytics components for the captured energy data. A particular challenge was to choose the right processing paradigms and technologies to cope with the high amount of sensors data. This section provides insights about the requirements and corresponding solution architecture that we used the project. In addition, we discuss the implementation selected analytics components (i.e. consumption prediction and power quality anomaly detection) in detail.

\subsection{Requirements and Solution Architecture}

The solution architecture for our testbed was primarily driven by three key requirements: (1) handling large data volumes, (2) handling high velocity data, and (3) flexibility regarding functional extensions. The first two requirements directly map to two of the three Vs that define big data (i.e. volume and velocity). The requirement for handling large data volumes is driven by the huge number of capture sensor measurements. Throughout the pilot we collected approximately 12 billion data points (about 1.2 million per household and day). This causes the need for technologies that can deal with this amount of data. The requirement for handling high velocity data is due to the need for real-time analysis of the incoming sensor streams. Applications that drive the need for real-time analysis are for instance load monitoring for end-users and consumption prediction for load balancing. End-users should have access to a live view of their consumption to get direct feedback about how their behavior influences energy usage. Consumption prediction for load balancing benefits from live data to drive short term adaptions of energy loads and support local balancing of the energy grid [16]. This caused the need for processing technologies that can deal with continuous processing over data streams. The third requirement - flexibility regarding functional extensions - stems from the nature of the testbed. The aim is to support exploratory data analysis and a growing number of analytics services. Hence, extensibility regarding the analytics functionality is a key concern for the solution architecture.

In order to address the above discussed requirements we adapted concepts of the lambda architecture for our solu- tion [10]. The lambda architecture provides a conceptual framework for the design big data systems. It defines the three layers (1) batch layer, (2) serving layer, and (3) speed layer to deal with different requirements independently and though dedicated technologies.

We use concepts of the batch layer to address the requirement for handling large data volumes. The batch layer persists all data and support parallelized processing in batches. It aims on scalability and high throughput at the cost of short response times. Our testbed persists all sensor data in a master data set that serves at input for distributed batch processing in a computer cluster. It allows exports as plain csv files and thereby supports a batch processing with a range of distributed processing system (e.g. Hadoop). We discuss an embodiment that we used in the pilot in [13].

We use concepts of the speed layer to address the requirement of handling high velocity data. The speed layer supports continuous processing in real-time but does not persist data. It keeps all data in memory and thereby enables realtime analysis. Input data from the sensors arrive at the system in a push based manner as read events and are abstracted though an adapter. This allows to connect to a range of stream processing systems (e.g. Apache Storm). We discuss specific embodiments that we developed in the pilot in [15].

To address flexibility for analytics extensions we follow the data modeling principles defined for the master data set in the lambda architecture. Specifically, we store sensor measurements in a fact based model, keep all raw measurements with a timestamp and refrain from updates as well as from aggregation or non-trivial preprocessing. This ensures that the maximum information value of sensor data is kept and future analytics are not constrained by design decision in the storage.

In the following subsections we discuss the implementation of two representative analytics components within the above framework.

\subsection{Power Quality Anomaly Detection}

Power quality anomaly detection is an analytics components that detect power quality issues in the low voltage grid. Specifically, it provides descriptive statistics about the deviation of the measured voltage from the target voltage. This deviation is subject to regulations and can impact the functioning of electrical devices or even cause damage. Consequently it is of high interest for grid operator to detect and understand voltage deviations within their grid. With our testbed we provide means to analyze this aspect of power quality on a new level of detail, i.e. the level of individual houses and even power outlets.

While the underlying mathematical operations are relatively simple, the analysis faces a significant big data challenge regarding data volumes (see [13]). For instance, in a sample experiment we analyzed voltage fluctuation of four households over the period of 1 month. This requires going over about 200 million measurements to check for voltage deviations. However, the use case does not pose any strong requirements on the response time for the query, making 


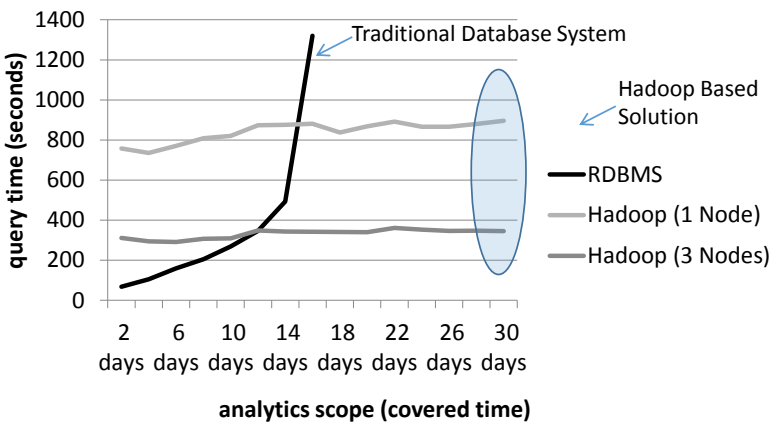

Figure 4: Comparison of query execution times with increasing database sizes

approached of the batch layer suitable for the implementation. An initial setup with batch processing on top of PostgreSQL failed to cope with the data volumes (see Figure 4). However, connecting the master data set with a Hadoop cluster and implementing power quality analysis as MapReduce jobs made the analysis feasible and proofed to be scalable (see Figure 4). Here, the map operation extracts the required subset of data points and computes the deviation, while the reduce jobs compute the aggregate descriptive statistics. Both operations run in batch mode and can be completed for the describe sample experiment in less than 7 minutes on a 3 node cluster.

\subsection{Consumption Prediction}

Accurate consumption prediction is a key enabler for load balancing in the local grid. The aim is to actively influence consumption so that local production (e.g. from solar panels) and local consumption match a closely as possible within a neighborhood. In order to influence consumption (e.g. though demand response mechanism) it must be known in advance. At least a few minutes lead time are desirable to invoke demand response measure.

We developed a prediction mechanism that uses the latest real-time information and household specific prediction models to make load predictions 15 minutes or an hour ahead. Our solution uses machine learning based prediction models that are trained for individual households and applied this models over live data streams. Experiments have shown, that this approach can improve prediction accuracy in the described setting [16]. However, it poses significant processing challenges. One key challenge is the training of individualized prediction models using the high volume of stored consumption measurements. Another key challenge is the continuous application of the models over real-time data streams.

We addressed this challenge by leveraging technologies of the batch layer and the streaming layer. We use the batch processing to train prediction models based on the collected historical data. This process covers feature extraction from the data records as well as the training process itself. The trained models are then loaded into the speed layer and remain in memory for real-time application. For real-time application, the input features are also extracted from the live data stream using in-memory operations. In a test imple- mentation following this paradigm we achieved continuous predictions with update rates of $0.5 \mathrm{~Hz}$ for 1000 households on a single machine (see [15] for details). The specific implementation was done with the CEP engine Esper for feature extraction in the stream [6] and Weka libraries for training machine learning models [7].

\section{END-USER SERVICES}

In this section we share experiences in providing web based end-user services on top of smart-home data, and report on challenges that we faced with common technology stacks for such a setup.

\subsection{Energy Consumption}

Within our testbed we provided a set of end-user services that allowed users to better understand their energy consumption. These services were a major driver for pilot users to participate in the project. The provided services include live statistics of device specific consumption values as well as long term statistics. In additions, the end-user services include functionality for remote control of actuators (i.e. switching smart plugs). Figure 5 shows screenshots of two sample services. Service 1 (Figure 5a) visualizes sensor data in real time and provides device specific as well as room specific consumption statistics. This includes a continuously updates live chart over the past few minutes as well as aggregate statistics that go back to the first measurement. In addition, the service allows remote switching of actuators. Service 2 (Figure $5 \mathrm{~b}$ ) provides device specific long term statistics. The statistics go back up to a month and in addition show a drill down of the consumption for the past week, the past day, and the current day. Despite its simplicity the service was very well perceived by end-users and turned out to be a key motivator for participation.

All services are accessible via a web interface. They are implemented following a typical design with a standard webserver on top of a relational database PostgreSQL (see Figure 1). The web based services were already launched early in the project when the components for big data processing were not available yet. However, this setup resulted in a set of challenges throughout the project.

A key challenge that we faced was that performance issues quickly arise when providing the described services on top of relational databases. Due to the high number of records (about 1.2 million records per house and day) maintenance operations like e.g. adding indices or exporting data very time consuming. Also, timely delivering query results required to tweak the database.

One specific challenge was providing the live chart in Service 1 that goes a few minutes back per sensor. This required to regularly query the database for measurements of a given sensor and a timestamp in a given range. Due to the high number of records, this query put a lot of load on the database. We solved this issues with an index on the timestamp that allowed to limit the scope of the query. Yet, the index on time did not solve all performance issues. In a monitoring component we regular check for the latest entry of each sensors. Since network failures (e.g. accidentally pulled network plug in a house) occasionally caused data gaps of several days. In such cases the monitoring query 


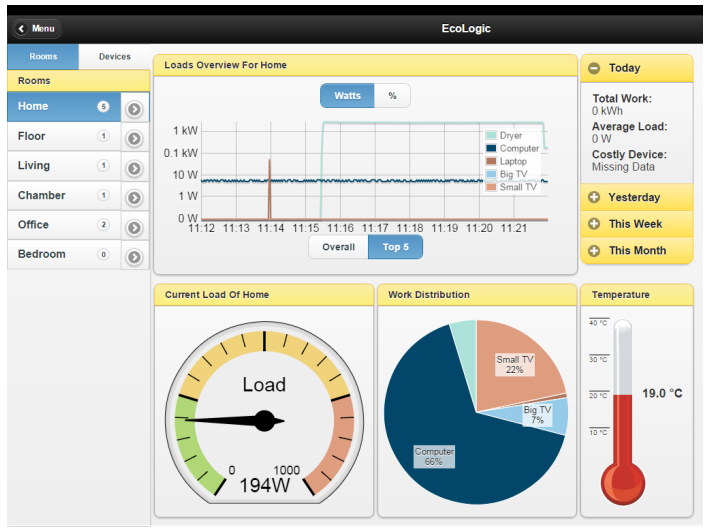

(a)

\begin{tabular}{|c|c|c|c|c|}
\hline \multicolumn{2}{|c|}{ \& Home } & \multicolumn{3}{|c|}{ onsumption Overview } \\
\hline Device & $\begin{array}{l}\text { Consumption } \\
\text { Today }\end{array}$ & $\begin{array}{l}\text { Consumption } \\
\text { Yesterday }\end{array}$ & $\begin{array}{l}\text { Consumption } \\
\text { Last Week }\end{array}$ & $\begin{array}{l}\text { Consumption } \\
\text { Last Month }\end{array}$ \\
\hline Computer & $0.04 \mathrm{kWh}$ & $0.08 \mathrm{kWh}$ & $5.6 \mathrm{kWh}$ & $12.61 \mathrm{kWh}$ \\
\hline Big TV & $0 \mathrm{kWh}$ & $0 \mathrm{kWh}$ & $1.34 \mathrm{kWh}$ & $4.77 \mathrm{kWh}$ \\
\hline Small TV & $0.11 \mathrm{kWh}$ & $0.23 \mathrm{kWh}$ & $3.02 \mathrm{kWh}$ & $8.93 \mathrm{kWh}$ \\
\hline Dryer & $0 \mathrm{kWh}$ & $0 \mathrm{kWh}$ & $0 \mathrm{kWh}$ & $0.08 \mathrm{kWh}$ \\
\hline Laptop & $0.02 \mathrm{kWh}$ & $0.04 \mathrm{kWh}$ & $0.27 \mathrm{kWh}$ & $0.73 \mathrm{kWh}$ \\
\hline
\end{tabular}

(b)

Figure 5: Service 1 with live statistics (a) and Service 2 with long term statistics (b)

needs to go back through millions of records and has a long response time.

Another challenge was the provisioning of long-term statistics. In Service 1 we show the device specific consumption since the start of recording. Ad-hoc computation of this statistic from the history of recorded load values is not feasible in a timely fashion (i.e. it would require computing the integral over the load curve with hundreds of millions of values). Here, we leverage a feature of the used smart plugs. These plugs provide an accumulated work value for the measured energy since installation. This value is transmitted with every measurement and we could use it directly in the statistics of Service 1. However, we still faced performance challenges for the more fine grained statistics of Service 2. Here we again leveraged the accumulated work value but the resulting queries still took several seconds due to the need for handling data gaps. The delay was too long for a satisfying user experience. Hence we implemented materialized view of the query results that were regularly updated with batch jobs.

\subsection{Privacy}

The smart metering ecosystem may facilitate a multitude of applications and value added services (VAS). Security and privacy are a major concern in this context as these applications and services directly affect users' everyday life and may collect a substantial amount of sensitive data.

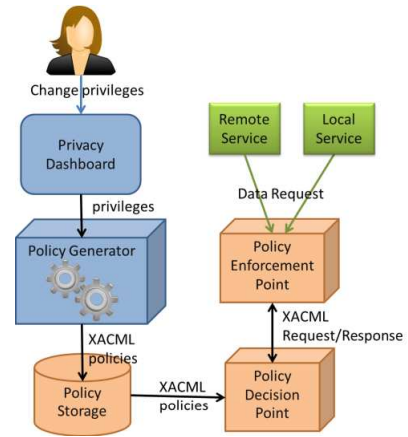

Figure 6: Privacy Model.

In order to enable these services new security and privacy solutions are required. The user needs simple-to-use mechanisms that provide a transparent view on all data that is collected and processed within such an ecosystem. The user should be in perfect control of which data is collected, how it is processed and which data is exchanged with which third parties. The PEC Privacy Dashboard is one of the components developed for the PEC project designed to define and control access to sensors. Fig. 6, depicts the components and their interaction.

The user interacts with a component called Privacy dashboard to review current access control rules and to modify as well as add rules. The user input from the privacy dashboard is transformed into statements in a given policy language. In order to make our approach as open as possible XACML [11] is used as the policy decision language, as its expressiveness allows full coverage of all necessary aspects for our application scenarios. These policy statements are then used to evaluate access requests from various applications. Specifically, the Policy Generator translates these PEC specific rules into generic XACML rules, policies, and policy sets and store them in the Policy Store. Service request data or data streams from an interface associated with the Policy Enforcement Point (PEP). The PEP translates data requests into XACML queries and forwards them to the Policy Decision Point (PDP). The PDP will load corresponding XACML policies from the store and attempt to decide on the request.

In the current version of the privacy dashboard shown in Fig. 7 deployed sensors and devices are presented to the user in a hierarchical fashion grouped first by the sensor type (e.g., motion, temperature, etc.) and within this group according to actual sensor location. While this layout reflects logical structure according to types of information requested from each application, it can easily be modified or adapted. Future adaptation might include information regarding the requested sampling rate of the service, or the sampling rate required for a particular service level.

For more details regarding the transformation into XACML policies refer to [4]. 


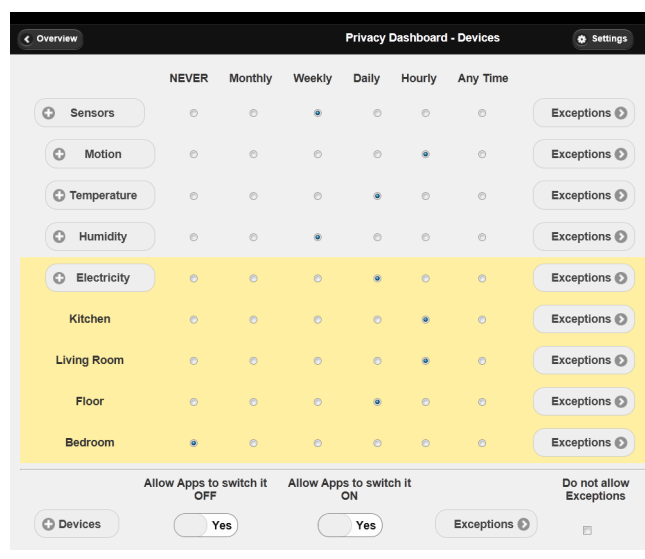

Figure 7: Privacy Dashboard.

\section{CONCLUSIONS}

In this paper we have provided results that were obtained thought a three year pilot with a cloud-based test bed for capturing and analyzing smart-home data. Our results include findings on real-world challenges in the application domain as well as technical solutions for addressing them. Among the challenges we found issues with data quality and data loss on several layers of cloud-based IoT solutions. In many cases, the issues are inherent to the application domain were we sense in home environments were users intentionally or unintentionally interfere with the operations of the system. With our solutions for sensor monitoring and deployment change detection we have shown ways how to address this issues. However, we see room for additional research and development efforts to expand the pool of techniques to cope with the data quality challenges. In particular, new techniques may be desirable when expanding the solution to new types of sensors.

Another set of key issues that we faced in the pilot was related to big data challenges. Our findings provide strong indication that classical relational database systems are hard use in given setup and dedicated big data technologies can provide better alternatives. We have shown solutions for a specific set of analytics that we have developed. Further research should expand the scope of considered analytics to validate and possibly adapt our suggested solutions for big data processing.

Overall, our work shows researchers as well as practitioners in the smart grid domain what challenges they have to expect when integration smart home technology. In addition, we describe solutions and approaches that can be followed and expanded to tackle such challenges.

\section{ACKNOWLEDGEMENT}

This work has been supported in part by the German Federal Ministry of Economics and Technology, project PeerEnergyCloud which is part of the Trusted Cloud Program.

\section{REFERENCES}

[1] Z. Alliance. Ieee 802.15. 4, zigbee standard. On http://www. zigbee. org, 2009.
[2] Z.-W. Alliance. About z-wave. Accessed at July 17th, 2014.

[3] C. Busemann, V. Gazis, R. Gold, P. Kikiras, A. Leonardi, J. Mirkovic, M. Walther, and H. Ziekow. Integrating sensor networks for energy monitoring with service-oriented architectures. International Journal of Distributed Sensor Networks, 2013, 2013.

[4] P. Ebinger, J. HernÃąndez Ramos, P. Kikiras, M. Lischka, and A. Wiesmaier. Privacy in smart metering ecosystems. In J. Cuellar, editor, Smart Grid Security, volume 7823 of Lecture Notes in Computer Science, pages 120-131. Springer Berlin Heidelberg, 2013.

[5] M. Erol-Kantarci and H. T. Mouftah. Wireless sensor networks for cost-efficient residential energy management in the smart grid. Smart Grid, IEEE Transactions on, 2(2):314-325, 2011.

[6] EsperTech. Esper event processing. On http://esper. codehaus. org, 2015.

[7] M. Hall, E. Frank, G. Holmes, B. Pfahringer, P. Reutemann, and I. H. Witten. The weka data mining software: an update. ACM SIGKDD explorations newsletter, 11(1):10-18, 2009.

[8] T. Hasan. Scalable distributed smart home monitoring and auto-configuration. Master's thesis, Technische Universität Darmstadt, Multimedia Communications Lab, 2014

[9] Linear. http://www.linear-smartgrid.be/. 2015.

[10] N. Marz and J. Warren. Big Data: Principles and best practices of scalable realtime data systems. Manning Publications Co., 2015.

[11] OASIS. eXtensible Access Control Markup Language 2 (XACML). Ed. Tim Moses., 2005.

[12] PeerEnergyCloud. http://www.peerenergycloud.de. 2015.

[13] M. Strohbach, H. Ziekow, V. Gazis, and N. Akiva. Towards a big data analytics framework for iot and smart city applications. In Modeling and Processing for Next-Generation Big-Data Technologies, pages 257-282. Springer, 2015.

[14] E. Williams, S. Matthews, M. Breton, and T. Brady. Use of a computer-based system to measure and manage energy consumption in the home. In Electronics and the Environment, 2006. Proceedings of the 2006 IEEE International Symposium on, pages 167-172. IEEE, 2006.

[15] H. Ziekow, C. Doblander, C. Goebel, and H.-A. Jacobsen. Forecasting household electricity demand with complex event processing: insights from a prototypical solution. In Proceedings of the Industrial Track of the 13th ACM/IFIP/USENIX International Middleware Conference. ACM, 2013.

[16] H. Ziekow, C. Goebel, J. Struker, and H.-A. Jacobsen. The potential of smart home sensors in forecasting household electricity demand. In Smart Grid Communications (SmartGridComm), 2013 IEEE International Conference on, pages 229-234. IEEE, 2013. 